\title{
Left Ventricular Pacing in Patients with Mechanical Tricuspid Valve Replacement
}

\author{
Sara Khodor $^{*}$, Shreya Mishra ${ }^{1}$, Dany Sayad ${ }^{2}$ and Anant Kharod ${ }^{2}$ \\ ${ }^{1}$ Division of Internal Medicine, University of South Florida, USA \\ ${ }^{2}$ Division of Cardiovascular Medicine, University of South Florida, USA
}

*Corresponding author: Sara Khodor, Division of Internal Medicine, University of South Florida, 2 Tampa General Circle, Tampa, FL 33606, USA

\section{Background}

In patients with mechanical tricuspid valve replacement (TVR), management of atrioventricular conduction abnormalities can be challenging given inaccessibility of the right ventricle (RV) with an endocardial pacing lead. While implantation of an epicardial lead may serve as an alternative; it requires invasive surgical placement and is not an ideal option in patients with prior thoracotomy. The development of specialized leads have made lead implantation via coronary sinus (CS) an attractive alternative for pacing in this patient population.

\section{Objective}

Discuss management approach for pacing in patients with mechanical tricuspid valve and important considerations indecision making.

\section{Case}

This case is of a 66-year-old female with a history of mechanical aortic, mitral, and tricuspid valve replacements in Spain in 1977 for endocarditis reportedly caused by a dental infection. She underwent repeat mechanical aortic valve replacement at Cleveland Clinic in 2012 for valve dysfunction and underwent mechanical mitral valve replacement for a large paravalvular leak in 2016. She was seen in clinic for evaluation of dizziness and bradycardia. ECG revealed atrial fibrillation with slow ventricular rate. Atrioventricular nodal blocking agents were held. Event monitor revealed chronic atrial fibrillation with symptomatic slow ventricular rate of thirty to fifty beats per minute with occasional four sec- ond pauses (Figure 1). After consideration of different management approaches, patient underwent implantation of single chamber pacemaker with placement of pacing lead within the CS (Figure 2) with satisfactory outcome on subsequent follow ups.

\section{Discussion}

Current guidelines recommend permanent pacemaker implementation for management of patients with atrial fibrillation with symptomatic slow ventricular response, as in the case of our patient [1]. However; when these patients have a mechanical tricuspid valve, the process of placing a pacemaker becomes challenging. The standard placement of an endocardial pacing lead is not recommended with mechanical TVR due to the risk of lead fracture at valve site and valve damage or failure. Similarly, removal of an existing endocardial lead is often necessary when tricuspid valve prosthesis is indicated. Leadless pacing also requires crossing the mechanical tricuspid valve for delivery and insertion of leadless pacemaker and thus is also not recommended.

A common alternative when the RV is inaccessible is placement of an epicardial pacing lead. Routine use of steroid-eluted epicardial leads designed to reduce inflammatory response and formation of fibrinous capsule has led to an improved 5 -years survival rates of $83 \%$ in comparison to nonsteroid-eluting leads with 5-year survival rates of $73 \%$, according to studies on pediatric population [2]. Steroid-eluted epicardial leads reportedly compare well with conventional endocardial systems; although no long-term lead comparisons studies have 


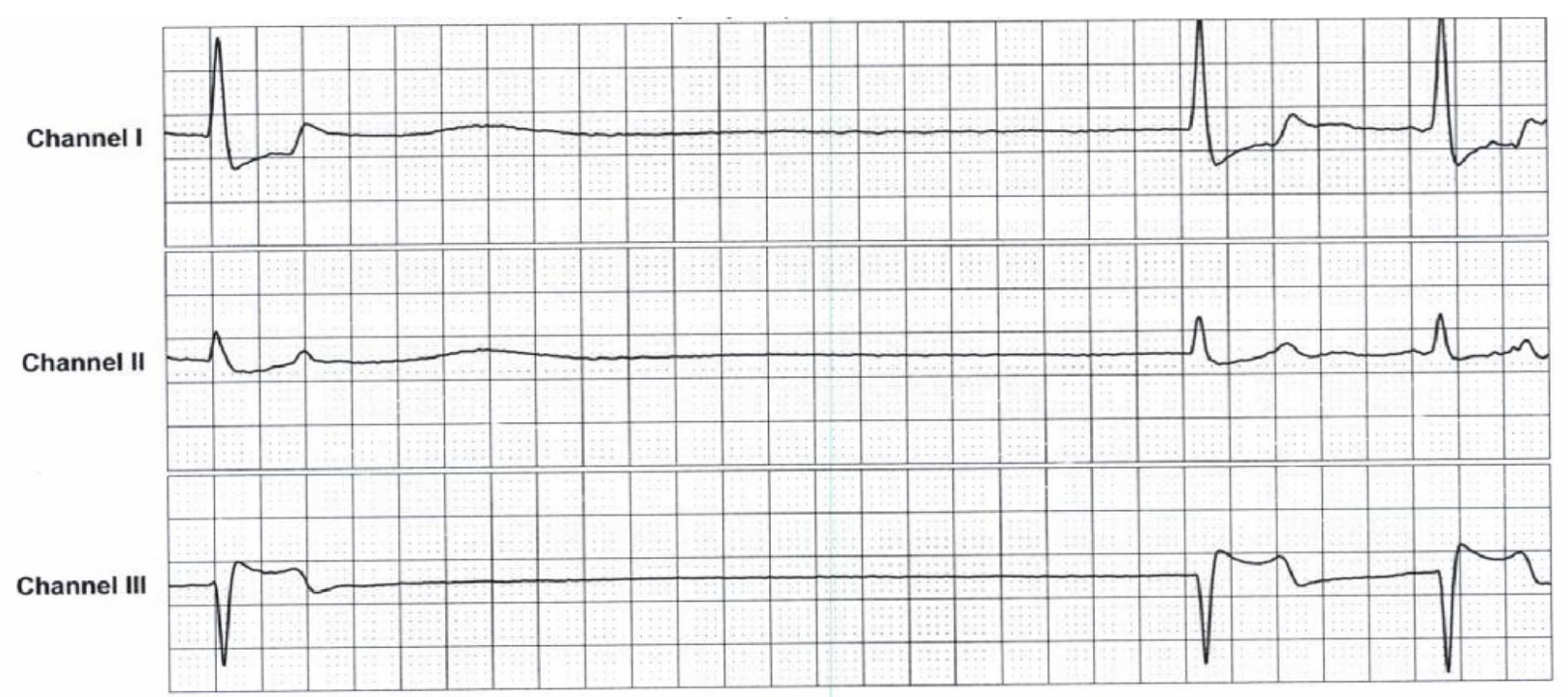

Figure 1: Event monitor demonstrating atrial fibrillation with 4.2 second pause.

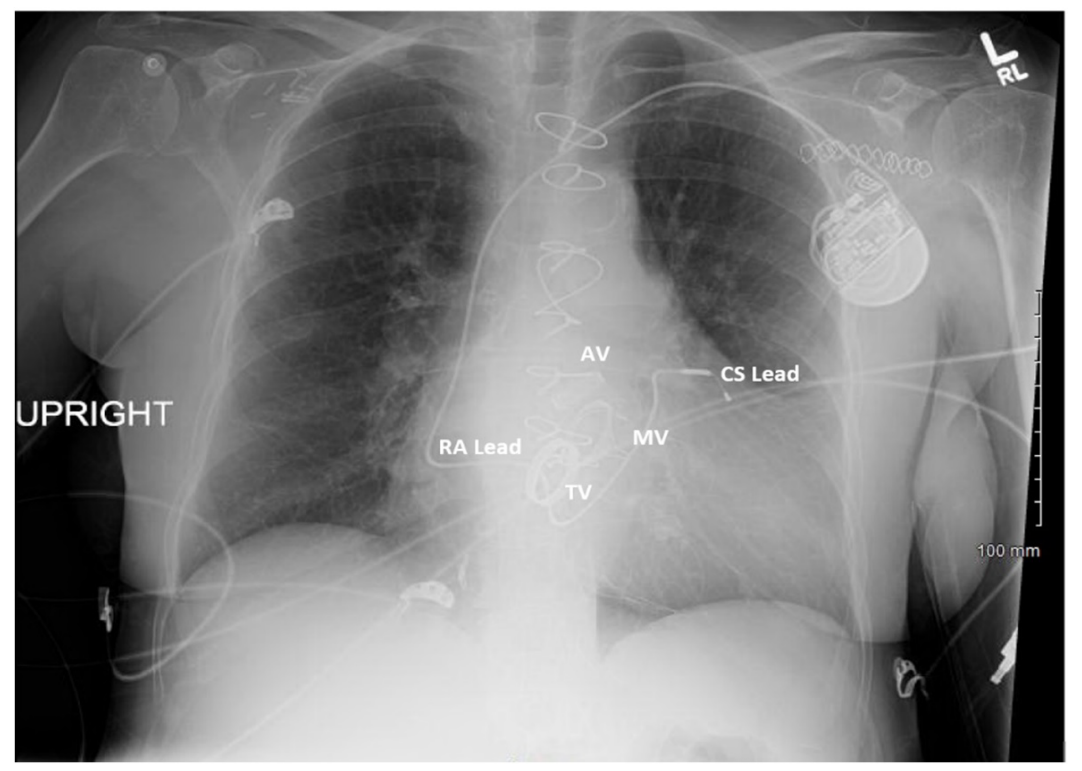

Figure 2: Chest X-ray with coronary sinus lead placement.

been performed to show this [2]. An important consideration in placement of an epicardial pacing lead is that it requires surgical approach which can pose technical difficulties and prolong hospital stays. In patients who have had multiple prior sternotomies or thoracotomies with resultant scar tissue and adhesion formation, as in the case of our patient, the risk of performing another surgery for epicardial lead placement can be associated with considerable morbidity.

In cases when the inaccessibility of the RV and the high risk of a repeat thoracotomy preclude placement of endocardial or epicardial pacing leads, it is imperative to consider an alternative approach for pacing via the CS. The idea of CS pacing was first described in 1970 and then in 1994 by Anagnostopoulos [3] and Bai [4] respectively and has since been used in patients with mechanical TVR and in congenital heart disease. This approach is not only minimally invasive but it also provides a stable position in the coronary vein, a reliable low threshold and adequate ventricular pacing. On the other hand, risks of CS pacing include diaphragmatic stimulation, coronary vein dissection, lead displacement, and infection [5].

Pacing via CS has been performed using conventional RV pacing lead [4] but posed greater procedural difficulty and high pacing threshold. The advancement in specialized leads with guiding catheters designed to allow easier access to the CS ostium and to the venous system facilitated left ventricular long-term pacing and transformed it into a more reliable and feasible option. Left ventricular pacing via CS may therefore be considered as an alternative for management of atrioventricular conduction abnormalities in patients with inaccessible RV. 


\section{References}

1. ACC/AHA Task Force (2008) ACC/AHA/HRS 2008 guidelines for device-based therapy of cardiac rhythm abnormalities. Circulation 117: E350-E408.

2. Cohen MI, Bush DM, Vetter VL, Tanel RE, Wieand TS, et al. (2001) Permanent epicardial pacing in pediatric patients. Circulation 103: 2585-2590.

3. Anagnostopoulos CE, Patel B, Fenn JE, Stansel HC (1970) Transvenous coronary sinus pacemaker. A new primary approach to heart block in patients with tricuspid prostheses. Ann Thorac Surg 9: 248-252.

4. Bai Y, Strathmore N, Mond H, Grigg L, Hunt D (1994) Permanent ventricular pacing via the great cardiac vein. Pacing Clin Electrophysiol 17: 678-683.

5. Alonso C, Leclercq C, Revaultd'Allonnes F, Pavin D, Victor $F$, et al. (2001) Six year experience of transvenous left ventricular lead implantation for permanent biventricular pacing in patients with advanced heart failure: Technical aspects. Cardiovascular Medicine 86: 405-410. 